\title{
Automatic Self-Calibration Of A Full Field-Of-View 3D n-Laser Scanner
}

\author{
Mark Sheehan, Alastair Harrison, Paul Newman
}

\begin{abstract}
This paper describes the design, build, automatic self-calibration and evaluation of a 3D Laser sensor using conventional parts. Our goal is to design a system, which is an order of magnitude cheaper than commercial systems, with commensurate performance. In this paper we adopt point cloud "crispness" as the measure of system performance that we wish to optimise. Concretely, we apply the information theoretic measure known as Rényi Quadratic Entropy to capture the degree of organisation of a point cloud. By expressing this quantity as a function of key unknown system parameters, we are able to deduce a full calibration of the sensor via an online optimisation. Beyond details on the sensor design itself, we fully describe the end-to-end extrinsic parameter calibration process, the estimation of the clock skews between the four constituent microprocessors and analyse the effect our spatial and temporal calibrations have on point cloud quality.
\end{abstract}

\section{Introduction and Related work}

3D laser range finding sensors are becoming both popular and important for mobile robotics, on account of their ability to produce accurate, dense surveys of a robot's surroundings. They may be used to produce detailed maps [1], to detect, classify and track items in the scene [2], and to recover the trajectory of the robot $[3,4]$. 3D laser range finders are usually of the 'actuated' variety: a relatively low-cost unit with a $2 \mathrm{D}$ field of view, mounted on an actuator which varies the scanning plane to cover a 3D volume. The popular but expensive Velodyne [5] laser range finder uses 64 lasers on a continuously rotating head to provide high bandwidth data over an elevational range of $26.8^{\circ}$. Our system employs three SICK LMS-151 scanning laser units - each

Mobile Robotics Group, Oxford University, \{mcs, arh, pnewman\} @ robots.ox.ac.uk. 


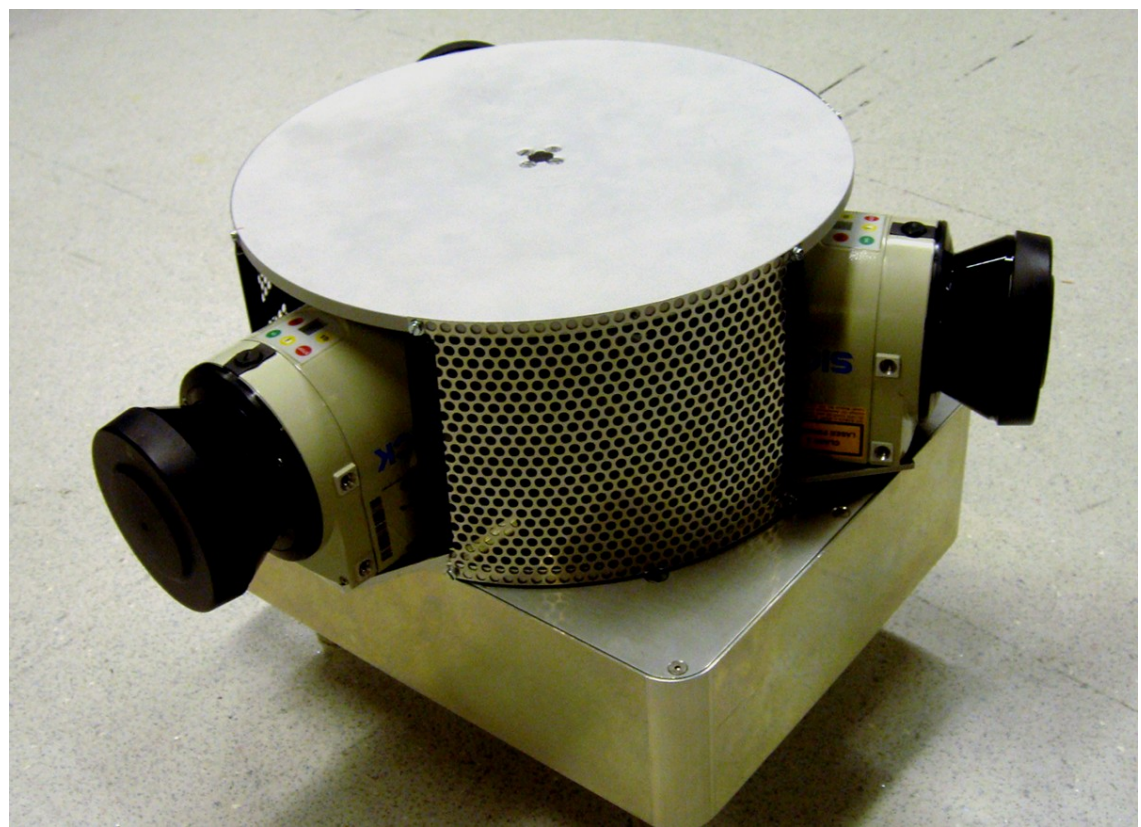

Fig. 1: An external view of our 3D scanner, which employs three SICK LMS151 laser rangers. These are $2 \mathrm{D}$ devices, with $0.5^{\circ}$ angular resolution over a $270^{\circ}$ sweep range, covered at a rate of $50 \mathrm{~Hz}$. Each unit makes 27,050 range measurements per second, so the total throughput of our system is 81,150 measurements per second. The LMS-151 units are mounted on a plate which rotates at speeds of up to $2.0 \mathrm{~Hz}$. A 12 line slip-ring supplies power and Ethernet to the rotating lasers, with a microprocessor taking encoder readings and acting as a motor controller for the plate.

with a nominal range of $50 \mathrm{~m}$ - mounted on a spinning plate. Figure 1 shows the arrangement, which allows for $360^{\circ}$ azimuthal coverage and almost full elevational coverage - the only unobservable volume being a cylinder through the axis of rotation, with a radius equal to the distance between the scanning planes of the lasers and the centre of the unit. Whilst the measurement throughput of our system does not match that of the Velodyne, the field of view is much greater and measurement fidelity is superior. However, the rotation rates of $1-2 \mathrm{~Hz}$ and lack of hardware synchronization between the lasers pose a significant challenge.

In the pursuit of quality 3D scans temporal and geometric calibration are essential. As an illustrative example, a time stamping error of just $15 \mathrm{~ms}$ (a typical clock accuracy error on a PC) on a laser rotating at a leisurely $1 \mathrm{~Hz}$, will result in just under $1 \mathrm{~m}$ of systematic error at a $10 \mathrm{~m}$ range. Of particular concern to us is the synchronisation of the laser range outputs with the sensor 
orientation measurements. This task requires the estimation of clock skews and offsets between all sensors and processors involved. Beyond temporal calibration we must also concern ourselves with the precise determination of the geometry of the system. Errors here also lead to a significant but avoidable performance degradation. In Section 2 we parameterise our system, outline the transformations used to convert raw sensor data to a world co-ordinate frame and outline the methods used for clock skew correction. In Section 3 we introduce a novel measure of point cloud quality which allows us to formulate an optimisation problem to automatically recover the correct geometric and temporal calibration parameters for our laser unit in Section 4. We provide both qualitative and quantitative results from our system in Section 5. Our experimental insights are given in Section 6.

\section{System Parameters}

\subsection{Kinematic Chain}

Consider a laser $L_{i}$ that takes measurements $\mathbf{Z}_{\mathbf{i}}=\left\{\mathbf{z}_{1} \ldots \mathbf{z}_{m}\right\}$ of a set of locations in the environment, $\mathbf{X}_{i}=\left\{\mathbf{x}_{1} \ldots \mathbf{x}_{m}\right\}$ as it is swept around by the plate. A measurement $\mathbf{z}_{j}=\left[r_{j}, \theta_{j}, \phi_{j}\right]^{T}$ consists of the range $r_{j}$, the laser's mirror angle $\theta_{j}$ and the plate position $\phi_{j}$. Our sensor model $h_{i}$ is $\mathbf{z}_{j}=h_{i}\left(\mathbf{x}_{j} ; \boldsymbol{\Theta}_{i}\right)$, where $\boldsymbol{\Theta}_{\mathbf{i}}=\left[\lambda_{i}, \tau_{i}, \alpha_{i}\right]^{T}$ is the set of extrinsic calibration parameters for laser $L_{i}$, explained in Figure 2. To estimate the position of the measured points given the measurements, we apply the inverse sensor model, resulting in a kinematic chain:

$$
\begin{aligned}
\hat{\mathbf{x}}_{j} & =h_{i}^{-1}\left(\mathbf{z}_{j} ; \boldsymbol{\Theta}_{i}\right) \\
& =\mathbf{R}_{\mathbf{z}}\left(\phi_{j}+\lambda_{i}\right) \mathbf{T}_{\mathbf{x}}\left(\tau_{i}\right) \mathbf{R}_{\mathbf{z}}\left(\alpha_{i}\right) \mathbf{R}_{\mathbf{y}}\left(\frac{\pi}{2}\right)\left[\begin{array}{c}
r_{j} \cos \left(\theta_{j}\right) \\
r_{j} \sin \left(\theta_{j}\right) \\
0
\end{array}\right]
\end{aligned}
$$

Here $\mathbf{R}_{\{\mathbf{x}, \mathbf{y}, \mathbf{z}\}}$ and $\mathbf{T}_{\{\mathbf{x}, \mathbf{y}, \mathbf{z}\}}$ are respectively rotations about, and translations along a given axis. By amalgamating a series of measurements over a period of time we are able to produce a 3D point cloud, $\hat{\mathbf{X}}_{i}=\left\{\hat{\mathbf{x}}_{1} \ldots \hat{\mathbf{x}}_{m}\right\}$ for laser $L_{i}$. Pooling measurements $\mathbf{Z}=\left\{\mathbf{Z}_{1}, \mathbf{Z}_{2}, \mathbf{Z}_{3}\right\}$ from all three lasers produces our final point cloud, $\hat{\mathbf{X}}=\left\{\hat{\mathbf{X}}_{1}, \hat{\mathbf{X}}_{2}, \hat{\mathbf{X}}_{3}\right\}$. 


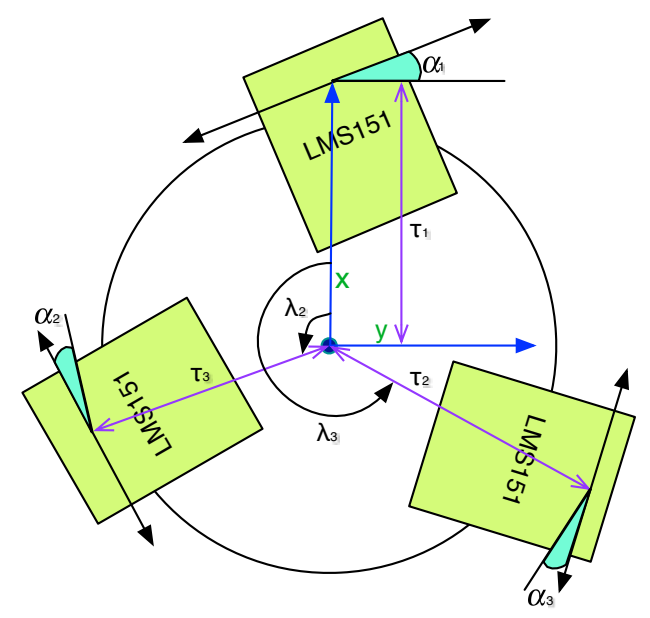

Fig. 2: The location of laser $L_{i}$ on the plate is defined by three parameters: $\tau_{i}$ is the distance of the beam origin from the centre of the plate, $\alpha_{i}$ is the angle between the laser's scanning plane and the tangent vector to the plate and $\lambda_{i}$ is the angle subtended between beam origins which lie along plate radials and $\lambda_{1}$ the beam origin for the first laser. For convenience we always set $\lambda_{1}=0$. These extrinsic parameters are automatically recovered using an optimisation procedure based on maximising point cloud quality.

\subsection{Learning the mapping between clocks}

The fidelity of the measured point cloud $\hat{\mathbf{X}}$ is highly dependent on the quality of the extrinsic calibration parameters and the accuracy of the plate rotation measurements. The latter being a function of the accuracy of the timestamps on both the plate encoder measurements and the individual laser measurements. Ideally we would have some function mapping laser measurement time stamps, $t_{j}$ to plate position measurements, so that $\phi_{j}:=\phi_{j}\left(t_{j}\right)$, but this would unrealistically require all devices to agree on the time. In fact, each LMS-151 time stamps its data using an internal clock; similarly, the microprocessor time stamps its readings of the plate encoder with its own internal clock. The situation is illustrated by Figure 3 .

The clocks in consumer grade equipment are notoriously temperature sensitive and there are no guarantees of accuracy. We performed an experiment where an LMS-151 was run continuously for 5 days and its time stamps recorded. At the end of the experiment, the clock had lost over 90 seconds compared to an accurate clock measuring UTC time. This is wholly inadequate for our needs. 
We could (as is typically performed) transfer all of the data to a central Hub PC and apply common time stamps upon receipt. For our application this would impose unacceptable noise, due to variable transport and buffering delays. Instead we choose to learn the mapping between the clocks on the different devices. We apply the algorithm of Moon et al. [6] to determine the relative frequency of each of the clocks relative to the clock of the Hub PC. Our implementation uses the efficient convex hull algorithm outlined in [7], which allows rapid online estimation of relative clock parameters. It operates by performing a linear programming optimisation on one-way offset measurements gathered from two clocks, which are separated by a variable delay data network. The algorithm can also recover the offset between the clocks, up to but not including the minimum transport delay, which is unobservable from one-way timing data alone.

After mapping device time stamps into Hub PC time stamps, we treat the unknown minimum transport delays as calibration parameters, $\eta_{i}$ represents the offset between laser time stamps and plate position time stamps for the $i$ th laser. Thus if we can determine the offsets, we can obtain correct plate position readings for every laser measurement from a laser $L_{i}$, via the function:

$$
\phi_{j}:=\phi_{j}\left(t_{j}+\eta_{i}\right)
$$

Sections 4 and 5 show how the unknown offsets can be determined by considering their effect on measured point cloud quality.

\section{A Measure of Point Cloud Quality}

Our motivation for finding a measure of point cloud quality was that with such a quantity, an optimization can be applied over the calibration parameters outlined in Section 2 resulting in a point cloud of high quality. Intuitively, we seek to find the calibration parameters which maximise the crispness of the point cloud.

We assume that our point cloud measurements $\hat{\mathbf{X}}=\left\{\hat{\mathbf{x}}_{1} \ldots \hat{\mathbf{x}}_{N}\right\}$ are drawn from an underlying distribution, $p(\mathbf{x})$ representing the probability of drawing a measurement from a given location, $\mathbf{x}$. To obtain an approximation of $p(\mathbf{x})$ we apply the Parzen Window [8] density estimation method. Using a Gaussian kernel centred on each data point, we are able to represent $p(\mathbf{x})$ as a Gaussian Mixture Model (GMM),

$$
p(\mathbf{x})=\frac{1}{N} \sum_{i=1}^{N} \mathbf{G}\left(\mathbf{x}-\hat{\mathbf{x}}_{i}, \sigma^{2} \mathbf{I}\right)
$$

where $\mathbf{G}(\mu, \boldsymbol{\Sigma})$ is a Gaussian with mean $\mu$ and covariance $\boldsymbol{\Sigma}$. An isotropic kernel is used, where $\boldsymbol{\Sigma}=\sigma^{2} \mathbf{I}$, with $\sigma$ being our systems only fixed tuning parameter. 


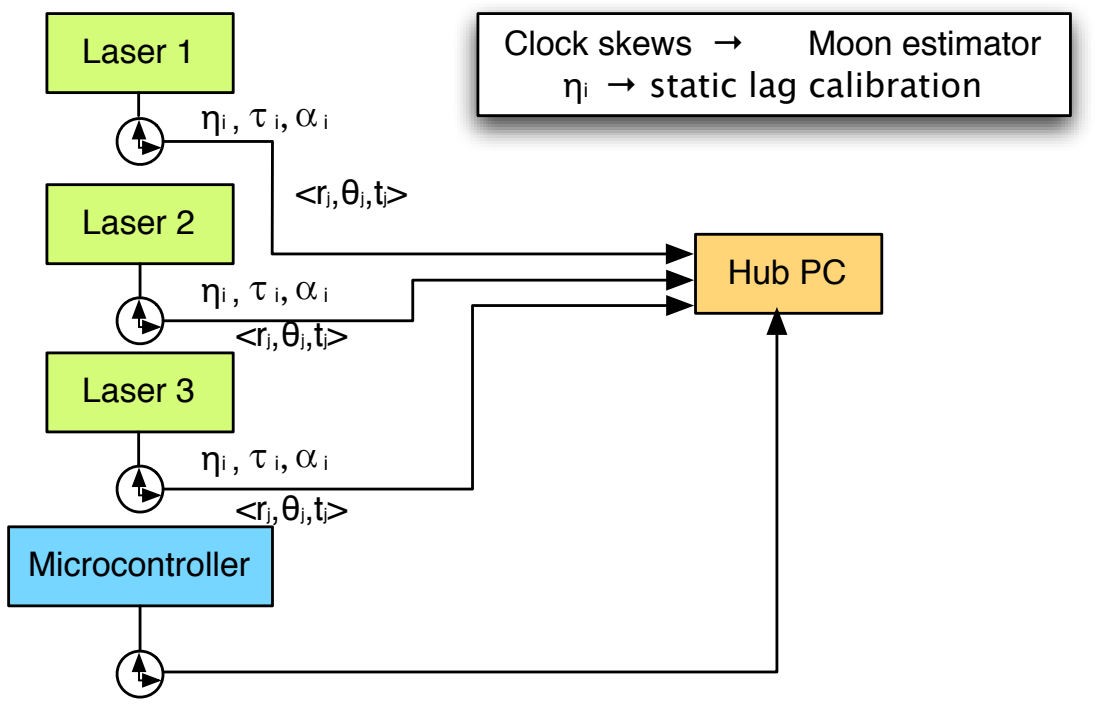

Fig. 3: A laser $L_{i}$ emits a stream of range $r_{j}$ and beam angle $\theta_{j}$ measurements with time stamp $t_{j}$, according to a clock $C_{i}$ within the laser. Each laser clock is characterised by a skew, relative to the clock on the hub PC and an offset $\eta_{i}$ relative to the clock of the microcontroller. The extrinsic calibration parameters $\tau_{i}$ and $\alpha_{i}$ are backed out from an analysis of plate orientation data in conjunction with the laser range data. The solve order is as follows: The clock skews are found via the algorithm of Moon et al. [6], $\eta_{i}$ from our static lag calibration (using nominal values of $\tau_{i}$ and $\alpha_{i}$ ). Finally $\eta_{i}$ is used to produce a refined estimate of $\tau_{i}$ and $\alpha_{i}$ for each laser. These values are then used to evaluate $\lambda_{i}$.

The 'crispness' of the point cloud can now be linked to the entropy of $p(\mathbf{x})$. The more crisp the point cloud, the more 'peaky' the distribution will be. An entropy measure proposed by Rényi [9] turns out to offer an efficient way to quantify the compactness of a GMM distribution [10] (as well as being a useful tool in the field of point cloud registration [11]). The Rényi entropy $H_{R}$ of a stochastic variable $X$ with pdf $p(x)$ is defined as

$$
H_{R}[X]=\frac{1}{1-\alpha} \log \int p(x)^{\alpha} d x \quad \alpha>0, \alpha \neq 1
$$

The single free parameter $\alpha$ determines how event probabilities are weighted: high values of $\alpha$ approaching infinity consider only the highest probability events whereas lower values of $\alpha$ weight high and low probability events more equally regardless of their likelihood. For the case where $\alpha \rightarrow 1$, Equation 5 becomes the familiar Shannon Entropy measure [12]. For $\alpha=2$ we obtain 


$$
H_{R Q E}[X]=-\log \int p(x)^{2} d \mathbf{x},
$$

which is known as the Rényi Quadratic Entropy (RQE).

We substitute the Gaussian Mixture Model of Equation 4 into Equation 6 to give,

$$
\begin{aligned}
H_{R Q E}[\hat{\mathbf{X}}] & =-\log \int\left(\frac{1}{N} \sum_{i=1}^{N} G\left(\mathbf{x}-\hat{\mathbf{x}}_{i}, \sigma^{2} \mathbf{I}\right)\right)^{2} d \mathbf{x} \\
& =-\log \left(\frac{1}{N^{2}} \sum_{i=1}^{N} \sum_{j=1}^{N} \int G\left(\mathbf{x}-\hat{\mathbf{x}}_{i}, \sigma^{2} \mathbf{I}\right) G\left(\mathbf{x}-\hat{\mathbf{x}}_{j}, \sigma^{2} \mathbf{I}\right)\right) d \mathbf{x} .
\end{aligned}
$$

Noting that the convolution of two Gaussians can be simplified as,

$$
\int G\left(x-x_{i}, \Sigma_{1}\right) G\left(x-x_{j}, \Sigma_{2}\right) d x=G\left(x_{i}-x_{j}, \Sigma_{1}+\Sigma_{2}\right),
$$

we obtain a closed-form expression for the Rényi Quadratic Entropy of the GMM,

$$
H_{R Q E}[\hat{\mathbf{X}}]=-\log \left(\frac{1}{N^{2}} \sum_{i=1}^{N} \sum_{j=1}^{N} G\left(\hat{\mathbf{x}}_{i}-\hat{\mathbf{x}}_{j}, 2 \sigma^{2} \mathbf{I}\right)\right) .
$$

Equation 10 can be thought of as a measure of the compactness of the points in X with an information-theoretic provenance for which the only free parameter is $\sigma$. For the purposes of optimisation it is noted that log is a monotonic operator and the scale factor is unnecessary, so those terms are dropped to produce our cost function,

$$
E(\hat{\mathbf{X}})=\sum_{i=1}^{N} \sum_{j=1}^{N} G\left(\hat{\mathbf{x}}_{i}-\hat{\mathbf{x}}_{j}, 2 \sigma^{2} \mathbf{I}\right)
$$

which depends only on pairwise distances between measured points in $\hat{\mathbf{X}}$.

\section{System Identification}

Substituting the inverse sensor model of Equation 1 into Equation 11 whilst compensating for the time lag offset corrections of Equation 3 allows the cost function to be written in terms of the extrinsic calibration parameters, $\boldsymbol{\Theta}=\left[\boldsymbol{\Theta}_{1}^{T}, \boldsymbol{\Theta}_{2}^{T}, \boldsymbol{\Theta}_{3}^{T}\right]^{T}$ and time stamp offset parameters $\mathbf{H}=\left[\eta_{1}, \eta_{2}, \eta_{3}\right]^{T}$, giving the cost function that we seek to optimise as: 


$$
E(\boldsymbol{\Theta}, \mathbf{H} \mid \mathbf{Z})=\sum_{i=1}^{N} \sum_{j=1}^{N} G\left(h^{-1}\left(\mathbf{z}_{i} ; \boldsymbol{\Theta}, \mathbf{H}\right)-h^{-1}\left(\mathbf{z}_{j} ; \boldsymbol{\Theta}, \mathbf{H}\right), 2 \sigma^{2} \mathbf{I}\right),
$$

Firstly we obtain the timing offsets $\mathbf{H}$. The error caused by incorrect lag values is proportional to the angular velocity of the plate. For this optimisation it is sufficient to use measurements from a single beam angle $\theta_{-45^{\circ}}$ in the plane of the rotating plate. Fixing $\tau_{i}$ and $\alpha_{i}$ to nominal values, we use a quasi-Newton method to optimise over the cost function of Equation 12,

$$
\hat{\mathbf{H}}=\min _{\mathbf{H}}(E(\boldsymbol{\Theta}, \mathbf{H} \mid \mathbf{Z}))
$$

giving optimised values of static lag $\hat{\mathbf{H}}$. The optimisation of Equation 13 must be performed with data collected at different plate speeds. The larger the variation in the plate speeds, the better conditioned the calibration problem becomes.

Now the lasers are temporally calibrated we use our values of $\hat{\mathbf{H}}$ to find $\hat{\tau}_{i}$ and $\hat{\alpha_{i}}$.

Consider now a 2D point cloud consisting of measurements from laser $L_{i}$ from two opposing mirror angles $\theta_{-45^{\circ}}$ and $\theta_{135^{\circ}}$, both in the plane of the rotating plate. We optimise to obtain estimates of extrinsic parameters $\boldsymbol{\tau}$ and $\boldsymbol{\alpha}$, where $\boldsymbol{\tau}=\left[\tau_{1}, \tau_{2}, \tau_{3}\right]^{T}$ and $\boldsymbol{\alpha}=\left[\alpha_{1}, \alpha_{2}, \alpha_{3}\right]^{T}$,

$$
\hat{\boldsymbol{\tau}}, \hat{\boldsymbol{\alpha}}=\min _{\boldsymbol{\tau}, \boldsymbol{\alpha}}(E(\boldsymbol{\Theta}, \mathbf{H} \mid \mathbf{Z}))
$$

The use of two mirror angles is necessary as the use of an extra mirror angle provides additional geometry necessary for calculating $\hat{\tau}$ and $\hat{\alpha}$.

Next we seek the relative alignment $\boldsymbol{\lambda}$ where $\boldsymbol{\lambda}=\left[\lambda_{1}, \lambda_{2}, \lambda_{3}\right]^{T}$ between the beam origins of each of the lasers. We use our optimised values $\hat{\boldsymbol{\tau}}, \hat{\boldsymbol{\alpha}}, \hat{\mathbf{H}}$, with the same mirror angles $\theta_{-45^{\circ}}$ and $\theta_{135^{\circ}}$,

$$
\hat{\boldsymbol{\lambda}}=\min _{\boldsymbol{\lambda}}(E(\boldsymbol{\Theta}, \mathbf{H} \mid \mathbf{Z}))
$$

A final optimisation over all free geometric parameters is performed to refine the crispness of the point cloud. Using previously obtained values of $\boldsymbol{\Theta}, \mathbf{H}$ to seed the optimisation,

$$
\hat{\boldsymbol{\Theta}}=\min _{\boldsymbol{\Theta}}(E(\boldsymbol{\Theta}, \mathbf{H} \mid \mathbf{Z}))
$$

A system with $n$ lasers will have $3 n-1$ geometric parameters to optimise over. 


\section{Results}

\subsection{Calibration results}

Figure 4 shows two $2 \mathrm{D}$ point clouds gathered from a single $\theta_{j}$ whilst plate velocity was varied between $0-2 \mathrm{~Hz}$. One plot is generated assuming zero timing offset, $\eta_{i}=0$ and the other for optimal lag value $\eta_{i}=\hat{\eta}_{i}$ obtained from the optimisation of Equation 13.
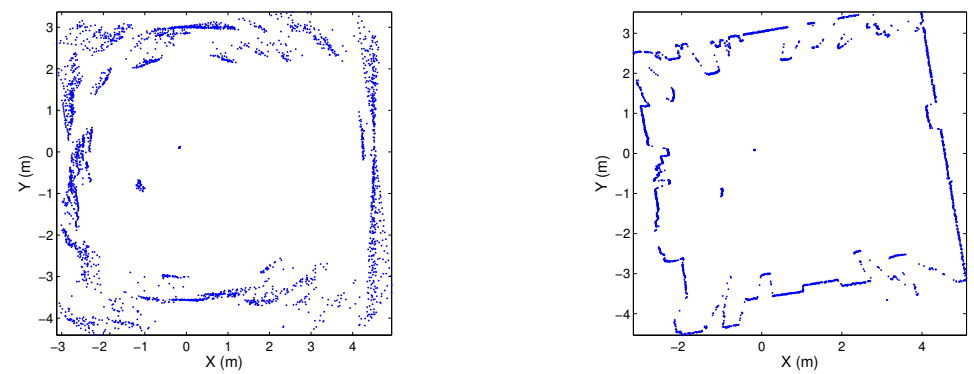

Fig. 4: Data from a single horizontal laser beam taken with the plate running between speeds of $0 \mathrm{~Hz}$ and $2.0 \mathrm{~Hz}$. The left hand image shows the points with an assumed lag of $\hat{\eta}=0 \mathrm{~ms}$ and the right hand image with $\hat{\eta}=38 \mathrm{~ms}$. The right hand image has a lower RQE measure.

Figure 5 shows a sampling of the cost surface generated in Equation 14. The cost surface can be seen to have a single (global) minimum with no local minima.

\subsection{Cost function verification}

We cannot numerically quantify the accuracy of the results obtained for the optimisations of Section 4 using real laser data as the ground truth values of our calibration parameters are unknown. To show that our calibration procedures estimate the true underlying parameters and to provide a quantifiable accuracy measure for our results, we have performed a series of Monte-Carlo simulations using synthetic laser data.

Our simulator generates measurements $\mathbf{z}_{\mathbf{i}}$ polluted by additive noise $N_{z} \sim N\left(0, \sigma_{z}^{2}\right)$ with $\left(\sigma_{z}=0.012 \mathrm{~m}\right)$ to match the statistical noise of the real laser measurements. The calibrations described in Equations 14 and 15 have been evaluated for this data with calibration parameters set to $\tau_{\text {true }}=0.20$, 


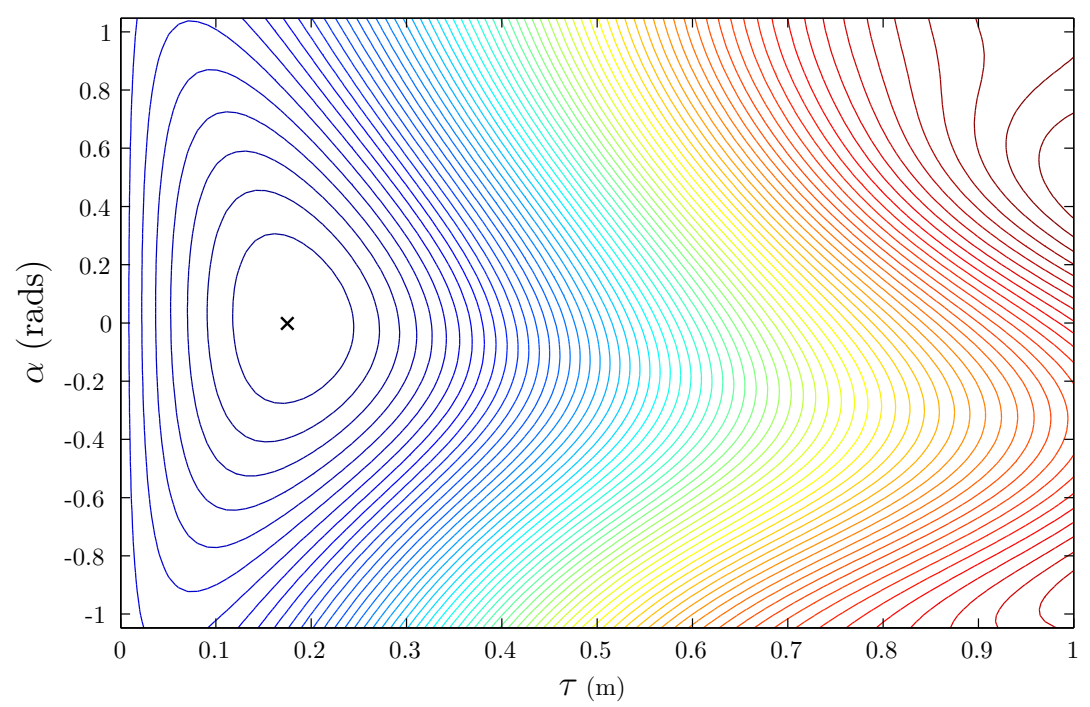

Fig. 5: Contour plot of cost surface $E(\Theta, \mathbf{H} \mid \mathbf{Z})$ generated using real laser data evaluated over a range of $\tau$ and $\alpha$ values. A cross is used to depict the global minimum.

$\alpha_{\text {true }}=0$ and $\lambda_{\text {true }}=0$ respectively. 1500 runs were performed with the simulated range data regenerated each time. Table 1 shows the results of these tests. We note that $\lambda$ optimised to within a range of $0.22^{\circ}$ for all 1500 runs despite being seeded with a worst case value of $\lambda=180^{\circ}$.

\begin{tabular}{|c|c|c|}
\hline Variable & Mean error & Standard Deviation \\
\hline \hline$\tau$ & $2.1 \mathrm{~mm}$ & $0.6 \mathrm{~mm}$ \\
\hline$\alpha$ & $0.06^{\circ}$ & $0.12^{\circ}$ \\
\hline$\lambda$ & $0.0009^{\circ}$ & $0.0322^{\circ}$ \\
\hline
\end{tabular}

Table 1: Deviance of results from ground truth values.

\subsection{Selection of free parameter $(\sigma)$}

To show how variation of free parameter $\sigma$ affects our estimates of calibration parameters we have generated simulated measurements $\mathbf{z}_{i}$ as in Section 5.2 to perform the optimisation of Equation 14 whilst varying $\sigma$ between 0.1 and 2. Figure 6 shows that estimations of $\tau$ and $\alpha$ become increasingly inaccurate 
with larger values of $\sigma$. Indicating that to optimise accuracy $\sigma$ should be set as low as possible, however looking at Figure 7 showing cost functions generated for Equation 13 using real data, we see that as $\sigma$ decreases the cost function becomes more 'peaky' until the extreme case of $\sigma=0.001$ where local minima are introduced to the cost function rendering it unsuitable for optimisation. It is recommended that $\sigma$ is initially selected to be significantly larger than the noise in the measurements, giving an optimisation over a cost function with a large basin of convergence, yielding an estimate of the calibration parameter, which should then be used to seed the optimisation with a value of $\sigma$ close to the measurement noise of the system.
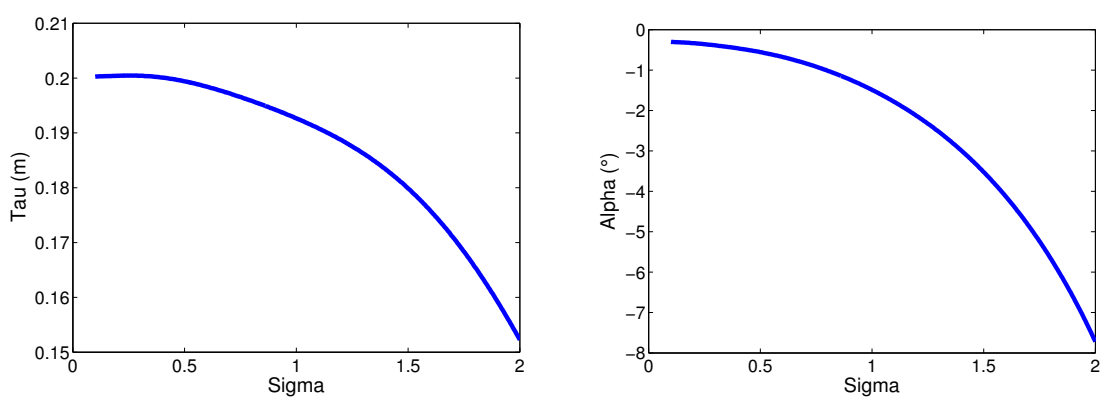

Fig. 6: Variation of calibration parameters $\alpha$ and $\tau$ with free parameter $\sigma$ where $\tau_{\text {true }}=0.2 \mathrm{~m}$ and $\alpha_{\text {true }}=0^{\circ}$

\subsection{Calibrated point cloud}

Having performed the optimisations listed in Section 4, we provide examples of point clouds generated from our system. Figure 8 shows data taken from an indoor office environment whilst rotating at velocities around $1 \mathrm{~Hz}$, Figure 9 shows a typical outdoor scene in an urban environment. Qualitatively the images appear crisp, as the RQE measure has been optimised over all extrinsic calibration parameters for each point cloud. 

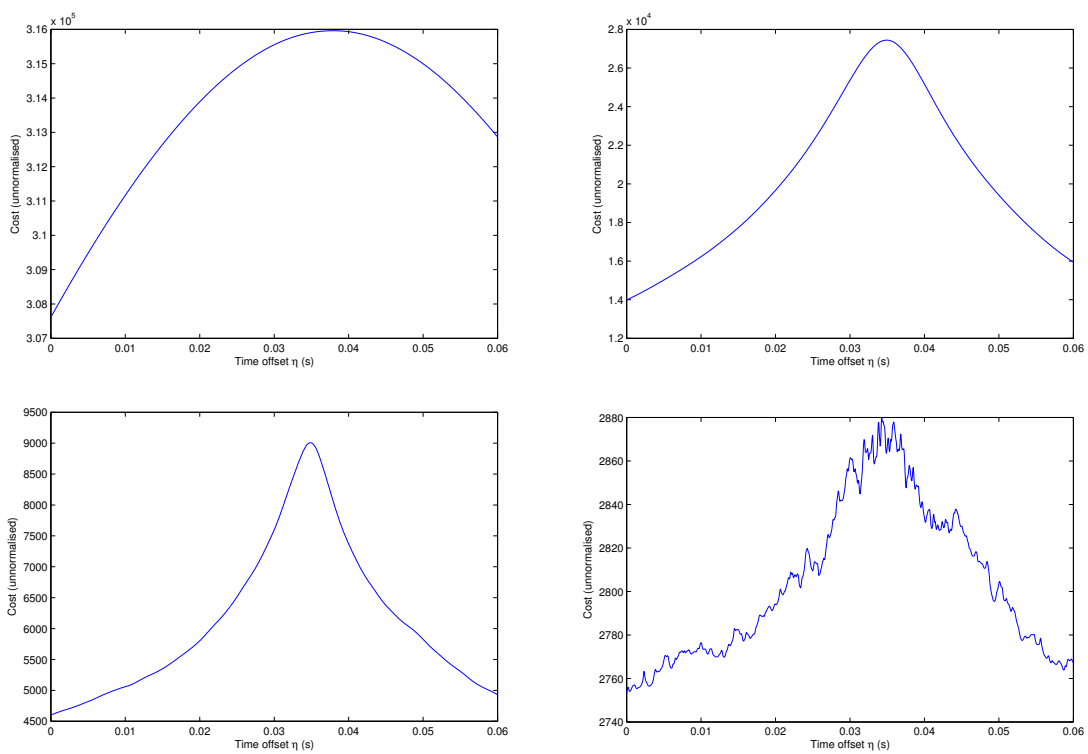

Fig. 7: The variation of the cost function of Equation 13 with free parameter $\sigma$ for real laser data. Using sigma values of, top left 0.5 , top right 0.04 , bottom left 0.012 , bottom right 0.001 .

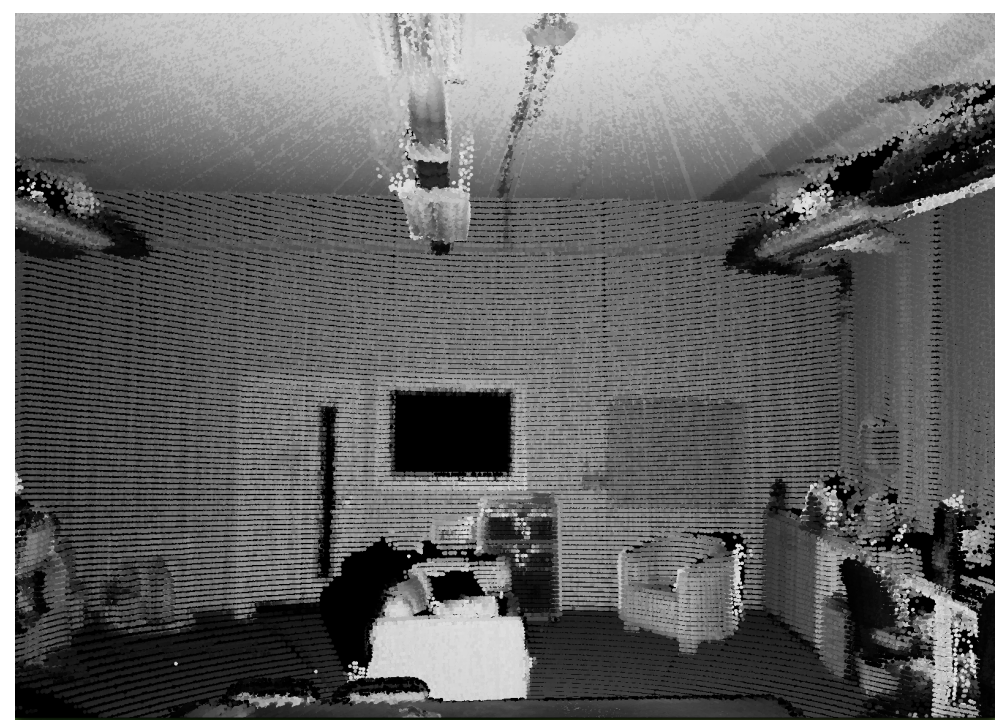

Fig. 8: A crisp point cloud of our lab generated using optimised values $\hat{\boldsymbol{\Theta}}, \hat{\mathbf{H}}$. Point colouring is based on laser reflectance values. This point cloud was achieved at a laser speed of $1 \mathrm{HZ}$. 


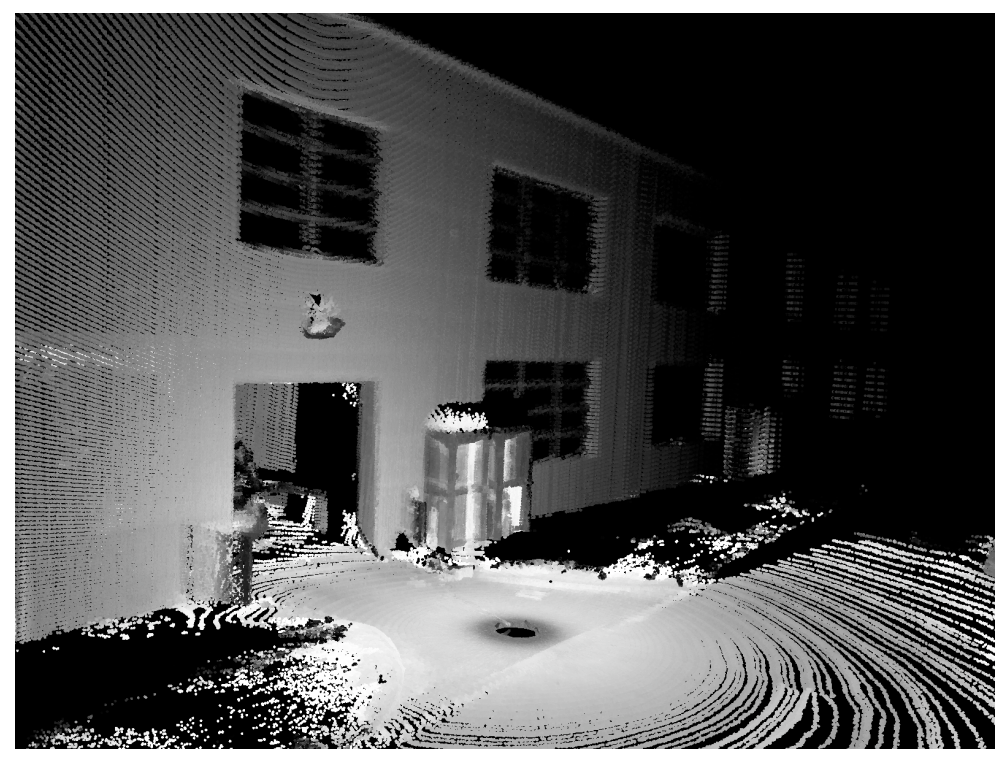

Fig. 9: A point cloud generated of a typical outdoor environment. Visualised a few metres from the laser scanner.

\section{Experimental insights}

In this paper we have given the specifications of our laser scanner, we have outlined a cost function based on Rényi Quadratic Entropy to measure the crispness of a point cloud and we have shown through the use of a MonteCarlo simulation that optimisation of this cost function over unknown system parameters yields quantifiably improved performance.

We note that spatial calibration is necessary only when the laser arrangement is changed relative to the plate and that our temporal calibration procedure only has to be performed once each time the unit is started. Whilst the cost function we use is $O\left(N^{2}\right)$, only a modest amount of data is required for the calibrations, thus optimisations remain tractable, resulting in a robust reconfigurable laser system.

\section{Acknowledgements}

This work has been sponsored by Guidance Ltd and by the European Commission under grant agreement number FP7-231888-EUROPA. 


\begin{tabular}{|c|c|}
\hline Symbol & Meaning \\
\hline \hline$L_{i}$ & The ith laser \\
\hline$r_{j}$ & The jth range measurement from a laser \\
\hline$\theta_{j}$ & The jth orientation measurement from the encoder \\
\hline$\phi_{j}$ & The distance from the rotational axis of the disc to the beam source of the ith laser \\
\hline$\tau_{i}$ & The geometric calibration parameters for the ith laser $\lambda_{i}, \tau_{i}, \alpha_{i}$ \\
\hline$\alpha_{i}$ & The angle between the scanning plane of the ith laser and the tangent to the plate \\
\hline$\lambda_{i}$ & The angle between the ith beam origin and the first beam origin along plate \\
\hline$\Theta_{i}$ & The timing offset between the ith laser and the encoder measurements \\
\hline$\eta_{i}$ & The Rényi Quadratic Entropy \\
\hline$H_{R Q E}$ & The only tuning parameter \\
\hline$E(\hat{\mathbf{X}})$ & \\
\hline$\sigma$ & \\
\hline
\end{tabular}

Table 2: List of parameters provided for reference.

\section{References}

1. A. Nuchter, K. Lingemann, J. Hertzberg, and H. Surmann, "6D SLAM with approximate data association," Advanced Robotics, 2005. ICAR '05. Proceedings., 12th International Conference on, pp. 242 - 249, 2005.

2. D. Steinhauser, O. Ruepp, and D. Burschka, "Motion segmentation and scene classification from 3D LIDAR data," Intelligent Vehicles Symposium, 2008 IEEE, pp. 398 403, 2008.

3. A. Harrison and P. Newman, "High quality 3D laser ranging under general vehicle motion," Robotics and Automation, 2008. ICRA 2008. IEEE International Conference on, pp. 7-12, 2008.

4. M. Bosse and R. Zlot, "Continuous 3D scan-matching with a spinning 2D laser," Robotics and Automation, 2009. ICRA '09. IEEE International Conference on, pp. 4312-4319, 2009.

5. Velodyne, "HDL-64E manual revision D," Apr 2008.

6. S. Moon, P. Skelly, and D. Towsley, "Estimation and removal of clock skew from network delay measurements," INFOCOM '99. Eighteenth Annual Joint Conference of the IEEE Computer and Communications Societies. Proceedings. IEEE, vol. 1, pp. 227-234 vol.1, 1999.

7. L. Zhang, Z. Liu, and C. H. Xia, "Clock synchronization algorithms for network measurements," INFOCOM 2002. Twenty-First Annual Joint Conference of the IEEE Computer and Communications Societies. Proceedings. IEEE, vol. 1, pp. 160- 169 vol.1, 2002.

8. E. Parzen, "On estimation of a probability density function and mode," The annals of mathematical statistics, vol. 33, pp. 1065-1076, Jan 1962.

9. A. Rényi, "On measures of entropy and information," Proceedings of the 4th Berkeley Symposium on Mathematics, Statistics and Probability, vol. 1, pp. 547-561, 1961.

10. J. Principe and D. Xu, "Information-theoretic learning using renyi's quadratic entropy," Proceedings of the 1st International Workshop on ..., Jan 1999.

11. Y. Tsin and T. Kanade, "A correlation-based approach to robust point set registration," Computer Vision-ECCV 2004, pp. 558-569, 2004.

12. C. Shannon, "A Mathematical Theory of Communication," Bell System Technical Journal, vol. 27, pp. 379-423, July,October 1948. 